Laboratory of Economics and Management Sant'Anna School of Advanced Studies Piazza Martiri della Libertà, 33 - 56127 PISA (Italy) Tel. +39-050-883-343 Fax+39-050-883-344

Email: lem@sssup.it Web Page: http://www.lem.sssup.it/

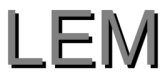

Working Paper Series

\title{
On the Irreconcilability of Pareto and Gibrat Laws
}

\author{
Giulio Bottazzi*
}

*Scuola Superiore Sant'Anna 


\title{
On the Irreconcilability of Pareto and Gibrat Laws
}

\author{
Giulio Bottazzi* \\ Center for the Analysis of Economic and Financial Dynamics \\ Scuola Superiore Sant'Anna
}

\begin{abstract}
If business firms face a multiplicative growth process in which their growth rates are independent from their sizes, then these sizes cannot be distributed according to a stationary Pareto distribution. At the same time, the Laplace distribution of growth rates cannot be easily reconciled with a Pareto distribution of firm sizes. Recent contributions, using formal arguments, seems to contrast these statements. We prove that the proposed formal results are wrong.
\end{abstract}

Key words: Firm Growth, Gibrat's Law, Power law distribution, Laplace distribution.

JEL Codes: L1,C1,D2

\section{Introduction}

Among the most popular, tested and contended hypotheses about the growth process of business firms one can, beyond any doubt, include the so called Gibrat's Law of Proportionate Effect, which postulates that firm growth rate is independent from firm size, and the Law of Pareto, which assumes a stationary power like behavior of the upper tail of the size distribution of firms.

* Scuola Superiore Sant'Anna, P.zza Martiri della Libertà 33, 56127, Pisa, Italy Tel.: +39-050-883365; fax: +39-050-883344;

Email address: bottazzi@sssup.it (Giulio Bottazzi). 
Recent publications propose to extend the number of these "Laws". The hypothesis that the (log) growth rates distribution robustly follows a Laplace, or doubleexponential, shape, originally proposed in Amaral et al. (1997), has received quite some attention in the related literature, and has been extensively tested in different countries and for different data (see the empirical review in Dosi (2007) and the theoretical investigation in Bottazzi and Secchi (2006)).

At the same time, in Fujiwara et al. (2003) it has been claimed that the joint distribution of firm sizes in two subsequent time steps is characterized by a "time symmetry" which makes the growth process reversible (a similar argument was previously put forward for the income distribution in Fujiwara et al. (2003)). Moreover, in Fujiwara et al. (2004), on the basis of the observed firm size time symmetry, it has been proposed that the Gibrat's Law of Proportionate Effect is consistent with the Pareto Law. Based on this result, Delli Gatti et al. (2005) suggested not only that these two Laws can be fulfilled at the same time, but that the Pareto behavior of the upper tail of the size distribution is in fact responsible for the observed Laplace shape of the growth rates distribution.

The mentioned statements in Fujiwara et al. (2003) and Delli Gatti et al. (2005) are in both cases derived by a formal proof starting from very general assumptions. It turns out that both these proofs contains analytical mistakes. As we will show through analogous formal arguments, not only the Laplace distribution of growth rates does not guarantee the contemporaneous fulfillment of both the Gibrat's and the Pareto's Laws, but none could. Indeed, the Law of Pareto and the Law of Gibrat cannot, in any respect, be reconciled: ignoring entry and exit dynamics, if firms face a multiplicative growth process in which their growth rates are independent from their sizes, then these sizes cannot be distributed according to a stationary Pareto distribution. Moreover, the claimed time-symmetry of the bivariate probability distribution of firms size is, by itself, uncompatible with the Gibrat's Law.

In the next Section we introduce a formal framework, largely borrowed from Fujiwara et al. (2004), that allows to express the previous statements in their whole generality. The, in Section 3, we will present the main points of this letter, that is we will discuss the relation between Gibrat's Law and the time symmetry of the size distribution put forward by Fujiwara et al. (2004) and the relation between Laplace growth rates distribution and Pareto's Law, commenting on Delli Gatti et al. (2005). The obtained results are finally summarized in the Conclusion. 


\section{Formal definition of properties}

Abstracting from precise economic definitions, let $S_{1}$ and $S_{2}$ be the size of a firm in two successive time steps. Let $p_{1,2}\left(S_{1}, S_{2}\right)$ stand for the joint probability density that a firm has size $S_{1}$ in the first time step and $S_{2}$ in the second. Consider the size return over the two periods $R=S_{2} / S_{1}$. By the simple rule of change of variables the joint probability density of the initial size $S_{1}$ and the return $R$ reads

$$
p_{1, R}\left(S_{1}, R\right)=S_{1} p_{1,2}\left(S_{1}, S_{1} R\right) .
$$

If $p_{1}\left(S_{1}\right)$ is the marginal distribution of the initial size, by Bayes rule one has

$$
p_{1, R}\left(S_{1}, R\right)=Q\left(R \mid S_{1}\right) p_{1}\left(S_{1}\right)
$$

where $Q$ stands for the conditional probability of a firm to grow at a rate equal to $R$ when its initial size is $S_{1}$. Analogously, one can write

$$
p_{1, R}\left(S_{1}, R\right)=P\left(S_{1} \mid R\right) p_{R}(R)
$$

where $p_{R}$ is the marginal distribution of return and $P$ the distribution of initial size conditional on the value of the return $R$.

The previous definitions allows us to express in rather general terms the properties mentioned above.

The Pareto Law states that the upper tail of the firms size distribution (or the distribution of a number of other economic variables, see Kleiber and Kotz (2003) for a partial account) approaches one with a power like behavior. In terms of probability density one has the following

Property 1 Pareto Law. If sufficiently large firms are considered, the probability density of their size decreases with some inverse power of the size itself

$$
p_{1}(x)=A x^{-\mu-1} \quad x>S_{\text {min }},
$$

where $\mu$ is known as the Pareto coefficient.

The second property, which is known as Gibrat's Law or "Law of proportionate effect" is probably the most famous and most tested hypothesis about the structure of the firms growth process (see Sutton (1997) for a summary review). One has 
Property 2 Gibrat Law. The firm's growth rate is independent on this size. That is

$$
Q\left(R \mid S_{1}\right)=Q(R) .
$$

The third property concerns the Laplace shape of the growth rates distribution: when the $\log$-return $r=\log (R)$ is considered the probability density behaves like a symmetric exponential. In terms of the return $R$ one has

Property 3 Laplace distribution The growth rate unconditional density $p_{R}(R)$ reads

$$
p_{R}(R)=\left\{\begin{array}{ll}
2 a R^{-a-1} & \text { if } \quad R>1 \\
2 a R^{a-1} & \text { if } \quad R \leq 1
\end{array},\right.
$$

where $a$ is a scale parameter.

The last property is strictly related to the degree of symmetry, in time, of the growth process. Roughly speaking, the idea is that if one reverse the "arrow of time" the growth process of firms look the same. Formally

Property 4 Detailed balance. The joint size distribution is symmetric, that is

$$
p_{1,2}\left(S_{1}, S_{2}\right)=p_{1,2}\left(S_{2}, S_{1}\right) .
$$

This means that the probability of a firm to jump from size $S_{1}$ to size $S_{2}$ over the considered period of time is equal to the probability of jumping, on the same period, from $S_{2}$ to $S_{1}$.

\section{Discussion}

Now that the properties we are interested in are formally defined, we can move to the main points of the present letter. In Fujiwara et al. (2004) it is stated that the contemporaneous fulfillment of Property 2 (Gibrat's Law) and Property 4 (detailed balance) implies the fulfillment of Property 1 (Pareto's Law). Actually, the following applies

Differently from what claimed in Fujiwara et al. (2004) one has the following 
Theorem 1 Properties 4 and 2 can never be satisfied at the same time

\section{PROOF.}

We will proceed with a reductio ad absurdum argument: we will assume that a probability density exists which fulfill both Properties and we will show that, in that case, it cannot be a probability density.

From (1) and Property 4 it is

$$
p_{1, R}\left(S_{1}, R\right)=S_{1} p_{1,2}\left(S_{1}, S_{1} R\right)=S_{1} p_{1,2}\left(S_{1} R, S_{2}\right)=\frac{1}{R} p_{1, R}\left(S_{1} R, R^{-1}\right) .
$$

Now according to Property 2 one has

$$
p_{1, R}\left(S_{1}, R\right)=p_{1}\left(S_{1}\right) Q(R)
$$

and

$$
p_{1, R}\left(S_{1} R, R^{-1}\right)=p_{1}\left(S_{1} R\right) Q\left(R^{-1}\right)
$$

so that from (8) it is

$$
p_{1}\left(S_{1}\right)=p_{1}\left(S_{1} R\right) \frac{1}{R} \frac{Q\left(R^{-1}\right)}{Q(R)} .
$$

Moreover, from the definition of marginal distribution and from (8) one has

$$
\begin{aligned}
Q(R)= & \int_{0}^{+\infty} d x p_{1, R}(x, R)=\int_{0}^{+\infty} d x \frac{1}{R} p_{1, R}\left(x R, R^{-1}\right)= \\
& \frac{1}{R^{2}} \int_{0}^{+\infty} d x p_{1, R}\left(x, R^{-1}\right)=\frac{1}{R^{2}} Q\left(R^{-1}\right)
\end{aligned}
$$

which, substituting in (9), gives

$$
p_{1}\left(S_{1}\right)=p_{1}\left(S_{1} R\right) R \text {. }
$$


This means that fixing any value of $c>0$ it is

$$
\lim _{x \rightarrow+\infty} p_{1}(c x)=\frac{1}{x}
$$

so that $p_{1}$ is not integrable and, consequently, cannot be a density function.

Notice that in Fujiwara et al. (2004) they use a different approach: they expand (9) around $R=1$ and put the first element of this expansion equal to zero. Of course this equation represents only a necessary conditions. Nevertheless the authors consider this condition sufficient, whence their mistake.

This Theorem shows that the hypothesis required by Fujiwara et al. (2004) can never be realized, so voiding the thesis of their argument.

The second point has to do with the claim, put forward in Delli Gatti et al. (2005), that the Pareto tail behavior is consistent with a Gibrat process characterized by a Laplace distribution of growth rates. On the contrary, one has the following

Theorem 2 Assume that the marginal distribution of $S_{1}$ satisfy property 1 . Then, if Properties 2 and 3 are satisfied, the marginal distribution of $S_{2}$ does not satisfy Property 1.

\section{PROOF.}

From Property 2 and Property 3 one has

$$
\begin{aligned}
p_{2}\left(S_{2}\right) & =\int_{0}^{+\infty} d S_{1} p_{1,2}\left(S_{1}, S_{2}\right) \\
& =2 a S_{2}^{-a-1} \int_{0}^{S_{2}} d S_{1} p_{1}\left(S_{1}\right) S_{1}^{a}+2 a S_{2}^{a-1} \int_{0}^{S_{2}} d S_{1} p_{1}\left(S_{1}\right) S_{1}^{-a}
\end{aligned}
$$

now if $S_{2}>S_{\text {min }}$, one can substitute (4) in the second term on the right hand 
side to obtain

$$
p_{2}\left(S_{2}\right)=2 a S_{2}^{-a-1} \int_{0}^{S_{2}} d S_{1} p_{1}\left(S_{1}\right) S_{1}^{a}+\frac{2 a}{a+\mu} A S_{2}^{-\mu-1} .
$$

Notice that when $S_{2} \rightarrow+\infty$ the integral in the first right hand term tends toward a constant, so that the resulting asymptotic behavior is a superposition of two powers, one with coefficient $\mu+1$ and one with coefficient $a+1$.

The previous Theorem states that, if at a certain point in time, the size distribution of firms follows the Law of Pareto, and these firms grow according to a Gibrat process with Laplace distributed shocks, then their distribution can no longer be a Pareto at a later point in time. The stationary Pareto distribution of firms size is incompatible with the Gibrat-Laplace process of growth.

More generally, in Delli Gatti et al. (2005) the authors claim that the Pareto size distribution is consistent with the Laplace growth rate distribution and suggest the following argument: in terms of $\operatorname{logarithm} s_{1}=\log \left(S_{1}\right)$ the behavior in (4) is equivalent to an exponential distribution. If both $s_{1}$ and $s_{2}$ are exponentially distributed random variables, then $r=s_{2}-s_{1}$, being the difference of two exponentials, is distributed according to a Laplace. In order for this argument to be valid, however, the variables $s_{1}$ and $s_{2}$ must be independent. This amount to say that

$$
p_{1,2}\left(S_{1}, S_{2}\right)=p_{1}\left(S_{2}\right) p_{2}\left(S_{2}\right)
$$

which is in contrast with any empirical evidence (also the ones reported in Delli Gatti et al. (2005)) and cannot be formally reconciled with Proposition 2. Indeed the expression of the conditional distribution of $R$ implied by (17) reads

$$
Q\left(R \mid S_{1}\right)=\frac{1}{S_{1}} p_{2}\left(R S_{1}\right)
$$

which depends on $S_{1}$. 


\section{Conclusions}

Many models have been proposed in the past to explain the empirically observed shape of the size distribution of firms and their growth process (see for instance the critical reviews and the original model proposed in Gabaix (1999)). In a way or another, they all violate at least one of the Properties presented in the previous Section. As we have seen, this is unavoidable. Indeed, we have proven that: first, the detailed balance condition proposed in Fujiwara et al. (2003) and Fujiwara et al. (2004) is inconsistent with a Gibrat growth process and, second, that the Gibrat process with Laplace growth rates is inconsistent with a Pareto size distribution. These proves are based on the analysis of the asymptotic behavior of the relevant distributions for large sizes, so they still hold true if one assumes that the previous Propositions are valid only above a certain size threshold. To a large extent, reconciling the different aspects of the growth process of firms (from entry/exit dynamics to Laplace growth rates distribution) still remains an open theoretical question.

\section{Acknowledgements}

Comments by Angelo Secchi and Mauro Gallegati helped in shaping this note. Research support by Scuola Superiore Sant'Anna (grant n. E6004GB) and by European Commission (CO3 NEST Pathfinder project, 6-th Framework program) is thankfully acknowledged

\section{References}

Amaral, L. A. N., Buldyrev, S.V., Havlin, S., Leschhorn, H., Maass, F., Salinger, M.A., Stanley, H.E. and Stanley, M.H.R. (1997) Scaling Behavior in Economics: I. Empirical Results for Company Growth, Journal de Physique I France, 7, 621-633.

Bottazzi, G., A. Secchi, (2006) Explaining the Distribution of Firms Growth Rates, Rand Journal of Economics, 37, pp. 234-263, 2006.

Delli Gatti D., C. Di Guilmi, E. Gaffeo, G. Giulioni, M.Gallegati and A. Palestrini (2005) A new approach to business fluctuations: heterogeneous interacting agents, scaling laws and financial fragility, Journal of Economic Behavior 6 Organization, 56, 489-512. 
Dosi, G. (2007) Statistical Regularities in the Evolution of Industries. A Guide through some Evidence and Challenges for the Theory, in Perspectives on Innovation Malerba, F. and S. Brusoni (Eds.), Cambridge University Press, Cambridge, U.K.

Fujiwara Y., W. Souma, H. Aoyama, T. Kaizoji and M. Aoki (2003) Growth and Fluctuations of personal income, Physica A, 321, 598-604.

Fujiwara Y., C. Di Guilmi, H. Aoyama, M. Gallegati and W. Souma (2004) Do Pareto-Zipf and Gibrat laws hold true? An analysis with European firms, Physica A, 335, 197-216.

Gabaix, X. (1999) Zipf's Law for Cities: an Explanation, The Quarterly Journal of Economics, 114, 739-767.

Kleiber, C. and Kotz, S. (2003) Statistical Size Distributions in Economics and Actuarial Sciences, New Jersey: John Wiley \& Sons.

Sutton, J. (1997) Gibrat's Legacy, Journal of Economic Literature, 35, 40-59. 